\title{
Influence of hydride purity on InP and InAlAs grown by chemical beam epitaxy
}

\author{
G.O. Munns ${ }^{\text {a }}$, W.L. Chen ${ }^{\text {a }}$, M.E. Sherwin ${ }^{\text {b }}$, D. Knightly ${ }^{\text {a }}$, G.I. Haddad ${ }^{\text {a }}$, L. Davis ${ }^{\text {a }}$ \\ and P.K. Bhattacharya ${ }^{\text {a }}$ \\ ${ }^{a}$ Center for High Frequency Micro-Electronics, Solid State Electronics Laboratory, 1135 EECS Building, The University of Michigan, \\ Ann Arbor, Michigan 48109-2122, USA \\ ' Sandia National Laboratory, Albuquerque, New Mexico 87185, USA
}

\begin{abstract}
It has generally been recognized that sources of the highest purity facilitate growth of InP and InAlAs with excellent optical and electrical characteristics. The mobility, photoluminescence linewidths, X-ray linewidths, impurity levels, and ultimately device results have been evaluated for two different hydride purity levels. It was found that each of the above characteristics was affected by improved arsine and phosphine purity. The $77 \mathrm{~K}$ mobility of InP increased by a factor of 2 and the InAlAs silicon doping efficiency increased by a factor of 5 . Accompanying improvement in device performance, notably the peak to valley ratio of resonant tunneling structures has been observed.
\end{abstract}

\section{Introduction}

One of the advantages of chemical beam epilaxy (CBE) is the ability to handle phosphorus and its related compounds. One of the key strengths therefore is the capability to produce epitaxial material lattice matched to InP. Only recently have high quality InAlAs and InP been grown by CBE. Several studies have shown that growth temperature, substrate misorientation, and phosphine cracking temperature are critical variables for the CBE epitaxial growth of InP with smooth surface morphology, high Hall mobility, good photoluminescence characteristics, and low background carrier concentration [1-3]. The origin of unintentional impurities found in InAlAs has previously been shown to arise from both the organometallic precursor and the hydride [4]. The incorporation of oxygen in InAlAs is known to have a deleterious effect on electrical and optical properties. To exploit the unique strengths of $\mathrm{CBE}$ it is necessary to appreciate the influence of impurities not only on bulk material properties of the films produced, but also on the concomitant effects on devices subsequently fabricated.
The development of trimethylamine alane (TMAA) catalyzed the successful growth of $\mathrm{Al}$ containing materials lattice matched to both $\mathrm{GaAs}$ [5] and InP [6,7]. In comparison to more conventional sources, TMAA lacks direct $\mathrm{Al}-\mathrm{C}$ bonds, decreasing the carbon incorporation while the oxygen reactivity is strongly reduced due to the coordinative saturation accomplished by the donation of the free electron pair of the trimethylamine (TMN) molecule to the $\mathrm{Al}$ atom of the aluminum hydride [8].

The recognized incorporation of impurities in InAlAs, specifically, motivated a series of steps toward their reduction in grown films. A correlation matrix design of experiment investigated the source of impurities in InAlAs [4]. In such a matrix, a value approaching 1 indicates a high degree of correlation with that variable. Table 1 shows that the source of $\mathrm{C}$ arose from the organometallic precusor (although it was not practical to completely decouple the group III flow from the $\mathrm{V}$ /III ratio) and the oxygen was found both in the arsine supply and the metalorganic. At the relative purity levels in the initial investigation, $\mathrm{AsH}_{3}: 2 \mathrm{ppm} \mathrm{H}_{2} \mathrm{O}$ and $2 \mathrm{ppm}_{2}$ 
and adduct purified organometallics (no detectable impurities by inductively coupled plasma), the primary oxygen contribution was determined to be from the hydride. Since the carbon incorporation could be controlled by the substrate temperature within an otherwise large parameter space, the primary concern became reduction of oxygen in the films. Two complementary approaches were used to accomplish this: (1) higher purity hydride sources were used for both arsine and phosphine and (2) commercially available hydride filters designed to remove water and oxygen from the gas stream were installed. The careful design of hydride filters had previously been shown to reduce the oxygen incorporation of $\mathrm{Al}$ containing films grown by gas source molecular beam epitaxy (GSMBE) [9].

\section{Experimental procedure}

Suitable (non-leaking) filters were installed in the system, an Intevac 1st generation CBE system utilizing hydrogen carrier gas delivery of trimethylindium, triethylgallium, and TMAA. The filter manufacturer specified a reduction of $\mathrm{H}_{2} \mathrm{O}$ and $\mathrm{O}_{2}$ below $10 \mathrm{ppb}$. Undiluted arsine and phosphine were used as the group $\mathrm{V}$ sources while the system was pumped by a $2200 \mathrm{l} / \mathrm{s}$ turbo. Hydrides were admitted to the reactor via a low pressure cracker. Careful attention to vacuum integrity was maintained, none the less OEM Viton sealed mass flow controllers were used despite the reported potential for impurity permeation of the elastomer [10]. Hydrides assayed at moisture and oxygen levels below $100 \mathrm{ppb}$ replaced sources with 2 ppm specifications. Identical growth conditions were maintained for each of the sources used; however, due to a limited number of hydride introduction lines, sequential switching between different assayed purities was not possible. Due to apparent abrasion of a diaphragm type valve integral to the hydride filter, it was impractical to isolate the effect of the purifier from the source material. Standard substrate preparation practices were common to all samples: volumetric 5:1:1 $\quad \mathrm{H}_{2} \mathrm{SO}_{4}: \mathrm{H}_{2} \mathrm{O}_{2}: \mathrm{H}_{2} \mathrm{O}$ followed by bromine:methanol etching. Films grown were grown under $\sim 10: 1$ group $V$ : group III conditions. Conventional solid source dopants (silicon) were evaporated from a Knudson style cell and while carbon contamination caused reduction in the efficiency of the cell after a period of approximately 40 runs, the doping calibration curves remained very stable (over $\sim 700$ runs), despite the neccessity of rather high running temperatures.

Bulk InP and InAlAs were grown with both source purities. Both types of films were subsequently evaluated by Hall measurement, low temperature photoluminescence, SIMS, double crystal X-ray diffraction, and Nomarski phase contrast microscopy. After calibration and characterization, device layers were grown, fabricated, and tested.

\section{Results and discussion}

\subsection{InAlAs Bulk}

The InAlAs grown with lower hydride purity had already demonstrated excellent device char-

Table 1

Linear correlation matrix for InAlAs secondary ion mass spectrometry (SIMS) results

\begin{tabular}{lllllll}
\hline & $T_{\text {sub }}$ & Group III & V/III & Oxygen & Carbon & Silicon \\
\hline$T_{\text {sub }}$ & 1 & 0 & 0 & 0 & -0.612 & 0.157 \\
Group III & & 1 & 0.703 & 0.740 & 0.609 & 0.371 \\
V/III & & & 1 & 0.926 & 0.112 & 0.304 \\
Oxygen & & & & & 0.166 & 0.367 \\
Carbon & & & & 1 & 0.220 \\
Silicon & & & & & 1 \\
\hline
\end{tabular}




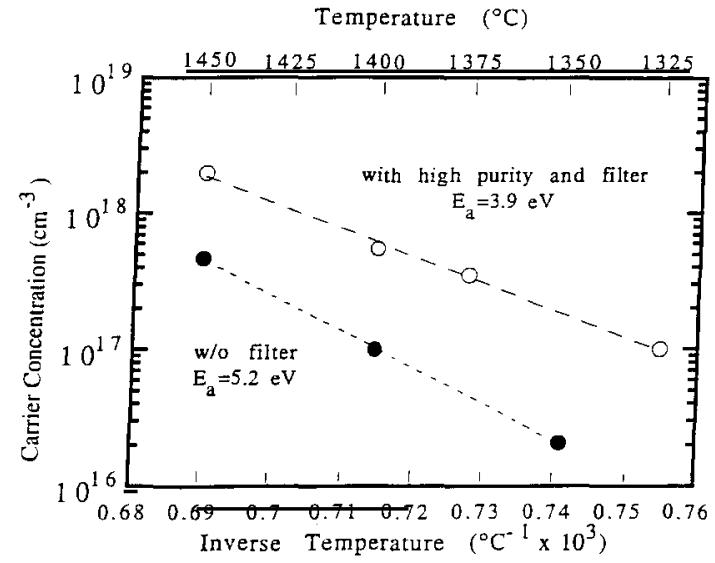

Fig. 1. Comparison of InAlAs:Si grown with and without filtering.

acteristics [11]. While initial SIMS measurements indicate no reduction in incorporated oxygen levels $\left(\sim(1-3) \times 10^{18} \mathrm{~cm}^{-3}\right)$, the silicon doping efficiency increased by a factor of 5 . This increase was not reflected in the n-type doping profiles of other material systems, and the measured background doping of the InAlAs did not increase. Fig. 1 compares the doping efficiency of silicon in InAlAs with and without filtering. The material grown with filtering shows an activation energy of $3.9 \mathrm{eV}$ in marked contrast to an activation energy of $5.2 \mathrm{eV}$ for unfiltered hydride. This is consistent with a reduction in silicon doping efficiency [12]; however, no similar reduction was observed in InGaAs or InP doped material. It is possible that the oxygen present in the films was due in this case to the metalorganic sources (specifically the TMAA which was $90 \%$ exhausted) and that this oxygen source was less efficient in inhibiting electrical activation of the donors than was the oxygen present in the hydride stream. This seems reasonable when one considers that the organometallics are known to form relatively involatile alkoxides and that these alkoxides may be incorporated in the growing films. Fig. 2 shows an example of InAlAs doped with silicon to $1 \times 10^{17}$ $\mathrm{cm}^{-3}$ and analyzed by SIMS. This sample exhibits close to unity electrical activation for the dopant. Double crystal X-ray diffraction (DCXRD) showed reliable lattice matching was maintained typically within $5 \times 10^{-4}$. Fig. 3 shows DCXRD

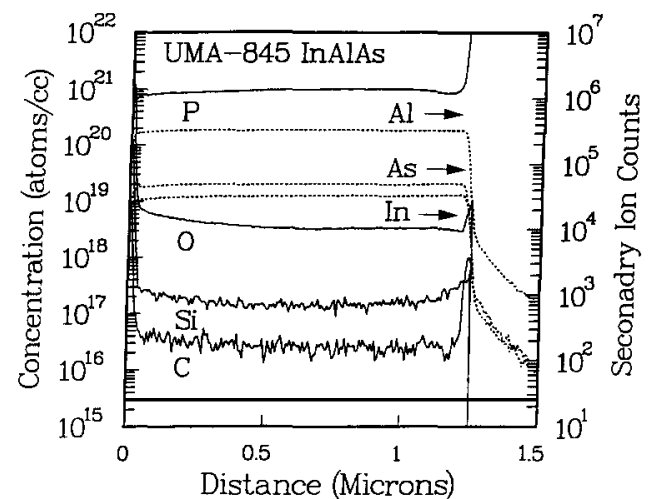

Fig. 2. Secondary ion mass spectra from InAlAs doped with silicon at $1 \times 10^{17} \mathrm{~cm}^{-3}$.

results for a layer grown with the improved purity hydride sources. It exhibits multiple pendellösung fringes and $|\delta a / a|$ of less than $5 \times 10^{-4}$ with no strain correction factor.

\subsection{InP Bulk}

Less expected were the bulk InP results which showed a rather dramatic improvement in the liquid nitrogen Hall mobilities. As has been previously reported $[3,4]$ there is a trade off between good surface morphology and Hall mobilities in InP. Prior optimization studies undertaken with the lower purity hydrides indicated a maximum

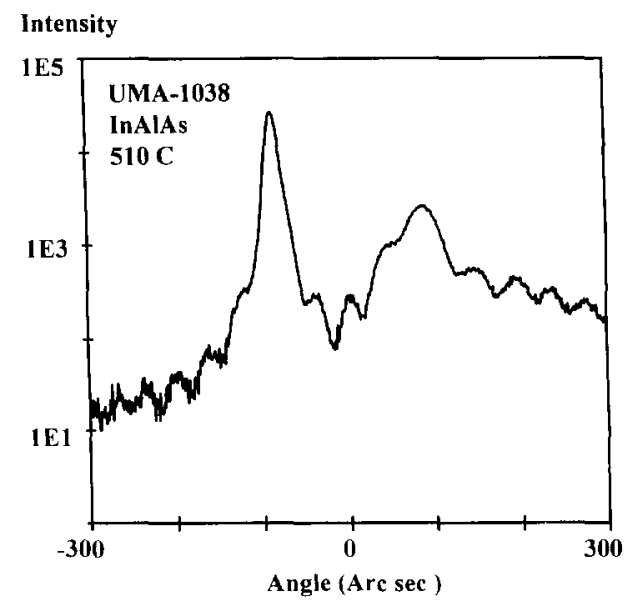

Fig. 3. Double crystal X-ray diffraction results for $0.63 \mu \mathrm{m}$ thick InAlAs. 
InP mobility of $4400 \mathrm{~cm}^{2} / \mathrm{V} \cdot \mathrm{s}\left(77,000 \mathrm{~cm}^{2} / \mathrm{V} \cdot \mathrm{s}\right)$. However, the conditions required to obtain these characteristics resulted in inferior surface morphology. A quiescent point which allowed a suitable compromise between surface morphology and electrical characteristics yielded $77 \mathrm{~K}$ mobilities of only $37,000 \mathrm{~cm}^{2} / \mathrm{V} \cdot \mathrm{s}$. Use of higher purity hydrides in conjunction with drying filters produced films with room temperature mobility of $5100 \mathrm{~cm}^{2} / \mathrm{V} \cdot \mathrm{s}\left(>90,000 \mathrm{~cm}^{2} / \mathrm{V} \cdot \mathrm{s}\right.$ at $\left.77 \mathrm{~K}\right)$ with associated background carrier concentration of $(1-3) \times 10^{14} \mathrm{~cm}^{-3}$ with excellent surface morphologies when grown under conditions identical to lower purity quiescent point conditions. Further improvement in Hall characteristics are predicted at higher substrate and lower hydride injector temperatures $[2,3]$ and there seems to be some increase in the satisfactory (smooth surface morphology) growth window. Initial $14 \mathrm{~K}$ photoluminescence results indicate that growth temperature and hydride injector temperature strongly influence the relative intensity of a donor-acceptor peak $\sim 31 \mathrm{meV}$ below the free exciton/bound exciton-donor complex (see fig. 4 for a representative PL spectra). DCXRD analysis showed a narrow (12 arc sec), symmetric single peak as in fig. 5. This is consistent with material grown under optimum conditions [3] as non-optimum conditions yield samples with broad, asymmetric X-ray characteristics.

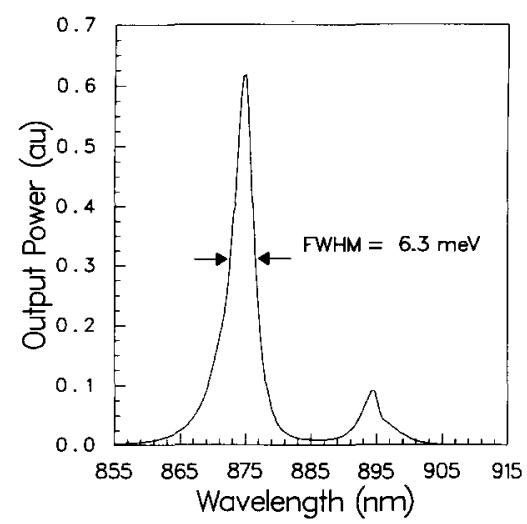

Fig. 4. Low temperature ( $18 \mathrm{~K}$ ) photoluminescence spectra of $3 \mu \mathrm{m}$ thick InP. Slits: $100 \mu \mathrm{m}$; lock-in: $25.0 \mathrm{mV}$.

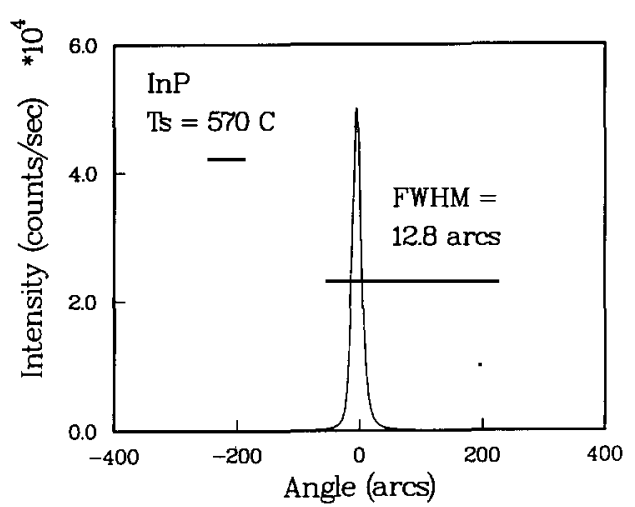

Fig. 5. Double crystal X-ray diffraction for $3 \mu \mathrm{m}$ thick InP.

\subsection{Devices}

A variety of device structures have been grown utilizing InP and InAlAs. Direct comparison of resonant tunneling structures grown with low purity and high purity hydrides showed that under identical conditions, the high purity hydrides resulted in peak to valley ratios twice that of structures grown with low purity hydrides [13]. Fig. 6 compares the results of two resonant tunneling transistors. The improvement in performance is attributed to a reduction of oxygen in the barrier and improved interface abruptness. This decrease in scattering centers yields a reduction in leakage current and valley current.

An InP channel modulation doped field effect transistor which utilizes both InP and InAlAs has also been grown. The Hall characterization of

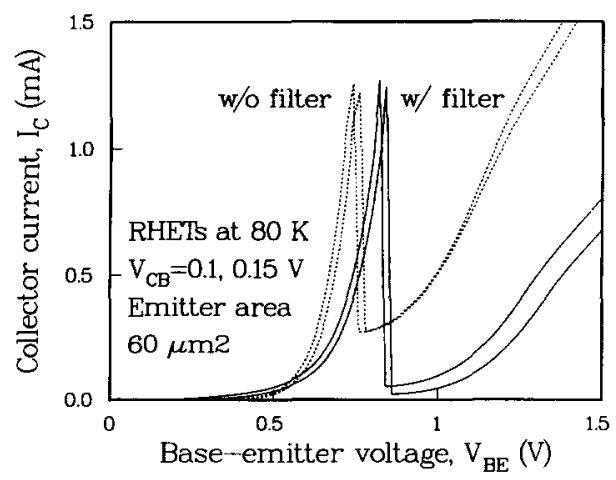

Fig. 6. Peak to valley ratio with and without hydride filtering. 
this device indicated higher room temperature and low temperature mobilities for a given carrier concentration for improved hydride purity. Specifically, the room temperature Hall effect mobility increased from 3800 to $4400 \mathrm{~cm}^{2} / \mathrm{V} \cdot \mathrm{s}$ for a sheet carrier concentration of $2 \times 10^{12} \mathrm{~cm}^{-2}$. However, device fabrication results have not been completed. Fig. 7 shows typical DC device char- acteristics for epi material grown without filtering.

Single and double heterojunction bipolar transistors (HBT) and resonant tunneling lasers grown with InAlAs emitters have also been fabricated in demonstration of the potential of high purity sources in the growth of minority carrier devices. These devices show reasonable DC characteris-
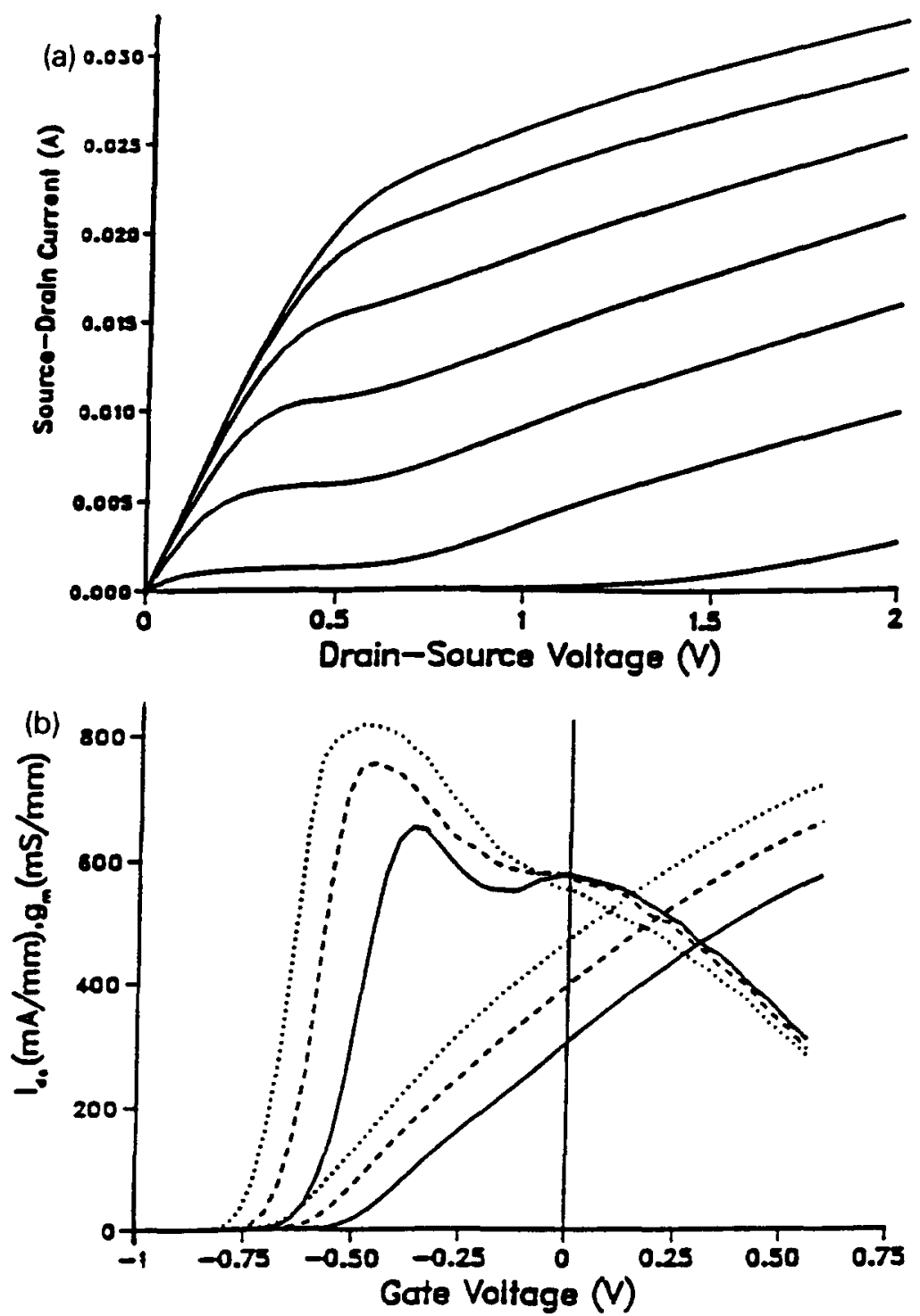

Fig. 7. (a) DC $I-V$ characteristics for InP HEMT grown without hydride filtering with a $0.1 \times 45 \mu \mathrm{m}$ gate. The maximum gate voltage is $0.6 \mathrm{~V}$, with a step size of $-0.2 \mathrm{~V}$. (b) Transconductance and $I_{\mathrm{ds}}$ versus gate voltage for same transistor. ( $-(-) V_{\mathrm{ds}}=1$ $\mathrm{V} ;(-\ldots-) V_{\mathrm{ds}}=1.5 \mathrm{~V} ;(\cdots \cdots) V_{\mathrm{ds}}=2 \mathrm{~V}$. 
tics [14]. Fig. 8 illustrates the $I-V$ characteristics for a typical double heterojunction transistor with a DC current gain of 50 and a breakdown voltage of approximately $8 \mathrm{~V}$ witness to the fact that the material is of excellent quality.

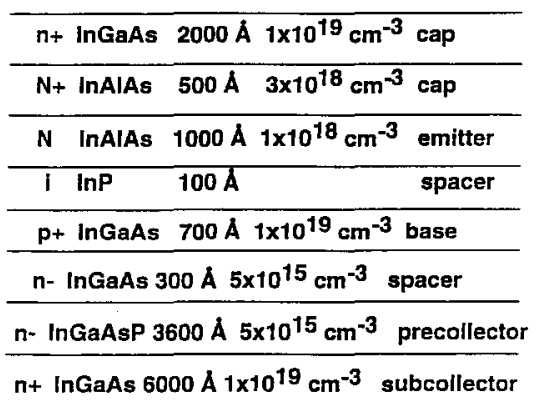

(a)

\section{Conclusion}

The influence of hydride source purity upon bulk InAlAs and InP has been shown to significantly affect electrical characteristics of these

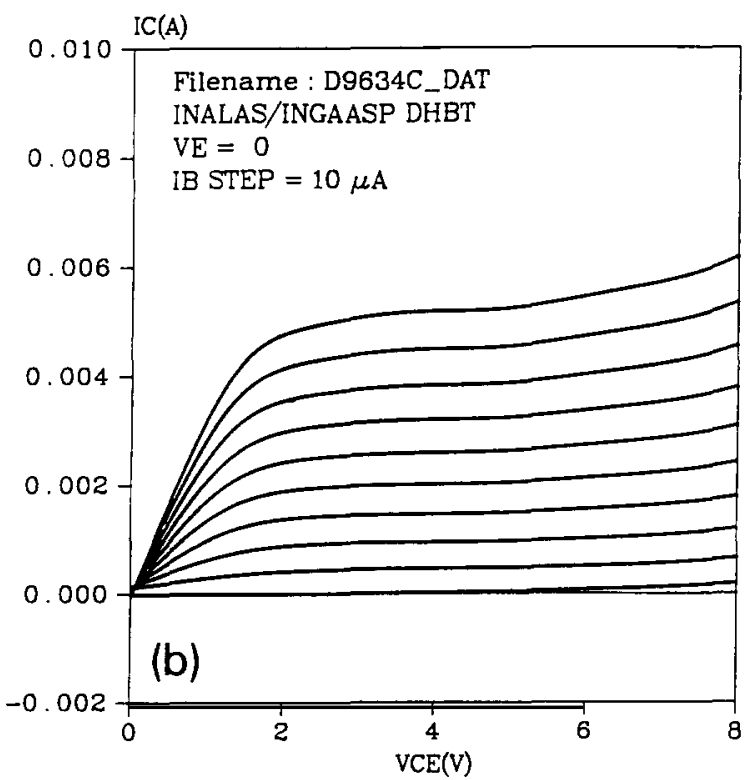

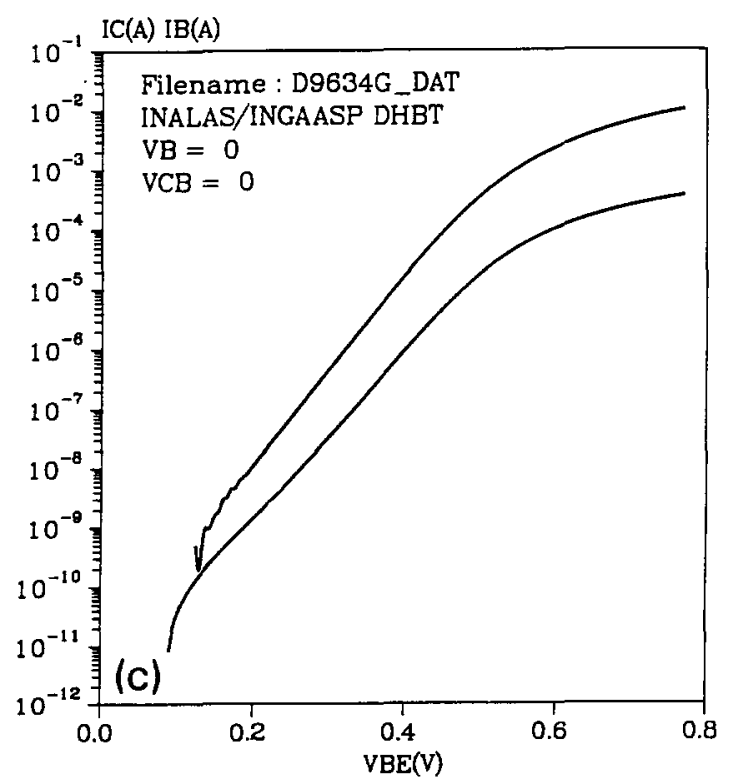

Fig. 8. (a) Structure of InAlAs/InGaAs/InGaAsP/InP double heterojunction bipolar transistor. (b) DC $I-V$ characteristics for DHBT. (c) Gummel plot of same transistor. 
films. A reduction in the oxygen found in phosphine not only results in dramatic improvements in low temperature Hall mobilities but it also enlarges the rather small window in which smooth surfaces are obtained. The ability to dope InAlAs with silicon from conventional solid sources is greatly improved when the oxygen content of the arsine utilized is reduced. The purity advantage enjoyed by devices has most clearly been demonstrated by the factor of 2 improvement in peak to valley ratio of resonant tunneling devices. The demonstration of minority carrier devices such as double heterojunction bipolar transistors illustrates the high quality of the films grown with filtering. Improvement in the Hall characteristics of InP channel HEMTs should directly impact device performance.

\section{Acknowledgements}

The authors would like to thank John Cowles for HBT fabrication and testing. This work was supported by ARO under the URI Program (Contract No. DAAL03-92-G-0109).

\section{References}

[1] H. Heineke, R. Höger, B. Baur and A. Miklis, Electron. Letters 26 (1990) 213.

[2] A. Rudra, J.F. Carlin, M. Proctor and M. Ilegems, J. Crystal Growth 111 (1991) 589.

[3] H. Rothfritz, G. Tränkle, R. Müller and G. Weimann, J. Crystal Growth 120 (1992) 130.

[4] M.E. Sherwin and G.O. Munns, J. Vacuum Sci. Technol. B 10 (1992) 943.

[5] C.R. Abernathy, A.S. Jordan, S.J. Pearton, W.S. Hobson, D.A. Bohling and G.T. Muhr, Appl. Phys. Letters 56 (1990) 2654

[6] J.L. Benchimol, X.Q. Zhang, Y. Gao, G. Le Roux, H. Thibierge and F. Alexandre, J. Crystal Growth 120 (1992) 312.

[7] G.O. Munns, M.E. Sherwin, Y. Kwon, T. Brock, W.L. Chen, D. Pavlidis and G.I. Haddad, J. Crystal Growth 127 (1993) 25.

[8] M. Kamp, F. König, G. Mörsch and H. Lïth, J. Crystal Growth 120 (1992) 124.

[9] M.J. Hafich, L.M. Woods, H.S. Kim, G.A. Patrizi and G.Y. Robinson, J. Crystal Growth 127 (1993) 995.

[10] J. Cerny (Intevac), private communication.

[11] G.O. Munns, M.E. Sherwin, T. Brock, G.I. Haddad, Y. Kwon, G.I. Ng and D. Pavlidis, J. Crystal Growth 120 (1992) 184.

[12] K. Beer, B. Baur, H. Heinecke and R. Treichler, J. Crystal Growth 120 (1992) 312.

[13] W.L. Chen, G.O. Munns, L. Davis, P.K. Bhattacharya and G.I. Haddad, J. Crystal Growth 136 (1994) 50.

[14] J.C. Cowles, W.L. Chen, G.O. Munns and G.I. Haddad, InP and Related Materials Conf., Paris, 1993. 